\title{
Notfällige Neurointerventionen, Covid-19 und Thorax-CT: SOP und Literaturübersicht
}

\author{
Horst Urbach ${ }^{1}$ (I) $\cdot$ Hendrik Janssen ${ }^{2} \cdot$ Jennifer Linn ${ }^{3} \cdot$ Titus Hoffmann $^{4} \cdot$ Stephanie Tritt $^{5} \cdot$ Werner Weber $^{6}$. \\ Martin Wiesmann ${ }^{7}$
}

Eingegangen: 21. April 2020 / Angenommen: 27. April 2020 / Online publiziert: 7. Mai 2020

๑) Springer-Verlag GmbH Germany, part of Springer Nature 2020

\section{Zusammenfassung}

Zielsetzung Analyse der „standard operating procedures“(SOP) von Bildgebung und Interventionen beim akuten Schlaganfall während der Coronavirus-disease-2019(COVID-19)-Pandemie. Bestimmung des Stellenwerts der Computertomographie (CT) des Thorax innerhalb eines multimodalen Schlaganfallprotokolls.

Methoden Über den E-Mail-Verteiler wurde ein Fragebogen an Mitglieder des Berufsverbands Deutscher Neuroradiologen e. V. verschickt. Stärken und Grenzen der Thorax-CT werden diskutiert.

Ergebnisse Es gingen Antworten aus 25 Abteilungen ein. Elf Abteilungen führen eine Thorax-CT durch, 3 bei jedem Patienten und 8 bei Verdacht auf COVID-19 aufgrund einer erhöhten Körpertemperatur, der Vorgeschichte des Patienten oder wenn Letztere nicht ausreichend erhoben werden kann. Vorläufige Daten weisen auf eine hohe Sensitivität und einen moderaten negativen prädiktiven Wert hin.

Schlussfolgerung Unterschiedliche SOP spiegeln eine gewisse Unsicherheit wider, ob eine Thorax-CT als Teil eines multimodalen Schlaganfallprotokolls durchgeführt werden sollte. Die Genauigkeit einer Low-dose-CT des Thorax kann noch nicht bestimmt werden.

Schlüsselwörter SARS-CoV-2 $\cdot$ Thrombektomie $\cdot$ Fragebogen $\cdot$ COVID-19 $\cdot$ Schlaganfall

\section{Acute Neurointerventions, Covid-19 and Chest-CT: SOP and Literature Review}

\section{Abstract}

Background and purpose To analyze standard operating procedures (SOP) of acute stroke imaging and interventions during COVID-19 pandemic with special emphasis on chest CT within a multimodal stroke protocol.

Methods A questionnaire was distributed via email to members of the Professional Organization of German Neuroradiologists (Berufsverband Deutscher Neuroradiologen e.V.).

Horst Urbach

horst.urbach@uniklinik-freiburg.de

1 Klinik für Neuroradiologie, Universitätsklinik Freiburg, Breisacher Str. 64, 79106 Freiburg, Deutschland

2 Institut für Neuroradiologie, Klinikum Ingolstadt, Ingolstadt, Deutschland

3 Institut für Diagnostische und Interventionelle Neuroradiologie, Universitätsklinikum Dresden, Dresden, Deutschland
4 Institut für Neuroradiologie, Universitätsmedizin Leipzig, Leipzig, Deutschland

5 Institut für Radiologie und Neuroradiologie, Wiesbaden, Deutschland

6 Institut für Diagnostische und Interventionelle Radiologie, Neuroradiologie und Nuklearmedizin, Universitätsklinikum Knappschaftskrankenhaus Bochum, Bochum, Deutschland

7 Klinik für Diagnostische und Interventionelle Neuroradiologie, Universitätsklinikum Aachen, Aachen, Deutschland 
Results Answers were received from 25 units: eleven of them acquire chest $\mathrm{CT}$, three in any patient and eight, when COVID-19 is suspected due to body temperature increase, patient's history or when the latter cannot be sufficiently obtained. Preliminary data indicate a high sensitivity and moderate negative predictive value.

Conclusion Different SOP reflect an uncertainty whether chest CT should be acquired as part of a multimodal stroke protocol. Accuracy of low dose chest CT cannot be determined yet. The strengths and limitations of chest CT are discussed.

Keywords SARS-CoV-2 $\cdot$ Thrombectomy $\cdot$ Questionnaire $\cdot$ COVID-19 $\cdot$ Stroke

\section{Hintergrund}

Bei Schlaganfall- und anderen Notfallpatienten kann das Ergebnis einer RT-PCR zum Nachweis von SARS-CoV-2, dem Erreger der COVID-19, aus einem Abstrich in der Mehrzahl der Fälle nicht abgewartet werden. Zudem ist eine Risikoanamnese (Fieber, respiratorische Infektion, Kontakt, ...) häufig nicht möglich. Ein solcher Patient wird also wie ein COVID-19-Verdacht betrachtet, auch wenn die Wahrscheinlichkeit, dass er mit SARS-CoV-2 infiziert ist, eher gering erscheint. Das wünschenswerte Szenario ist nun, dass der Patient die Bereiche Computertomographie (CT), Angiographie und Intensivstation wie ein COVID19-Patient durchläuft sowie Isolierung und Verdacht nach negativem RT-PCR-Ergebnis aufgehoben werden [1-3].

Dennoch sollte die Erkrankungswahrscheinlichkeit bei knappen Ressourcen (an Schutzmaterialien) zumindest abgeschätzt werden und vor der Durchführung des CT eine Fiebermessung erfolgen. Es wird auch diskutiert, ob ein aufgrund der Schlaganfalldiagnostik angefertigtes multimodales CT (natives CT, CT-Angiographie, CT-Perfusion) durch ein „Low-dose“-Thorax-CT ergänzt werden soll, das die Lungenabschnitte unterhalb der bereits mithilfe der CT-Angiographie abgedeckten Abschnitte erfasst, oder die CT-Angiographie bei kraniokaudaler Abtastung bis zu den Zwerchfellhälften zu „fahren“. Das Argument für ein Lowdose-Thorax-CT ist die in vielen Studien angegebene hohe Sensitivität in der Erfassung von COVID-19. Ob diese wirklich im Durchschnitt $85 \%$ beträgt (Tab. 1), muss jedoch kritisch hinterfragt werden, da durch die Untersuchung hospitalisierter und damit symptomatischer COVID-19-Patienten in den publizierten Studien ein Selektion-Bias vorgelegen haben könnte [4]. Argumente gegen den Einsatz des Low-dose-CT sind die niedrige Spezifität, der nur moderate negative prädiktive Wert und die zeitliche Abhängigkeit des Auftretens der CT-Veränderungen. Hieraus resultieren Empfehlungen von Fachgesellschaften, das Low-dose-CT nicht als Screeninguntersuchung zu verwenden [5-7]. Chinesische Organisationen plädieren für einen weitflächigen Einsatz [8] und Fachgesellschaften wie die Radiological Society of North America (RSNA) und die Fleischner Society „sehen“ einen gewissen Stellenwert des Low-dose-Thorax-
CT, wenn die RT-PCR nicht sofort verfügbar ist $[5,9]$. In einer eigenen Handlungsempfehlung wurde das Thema einer notfallmäßigen Einschätzung weitgehend ausgeblendet [10]; in dieser Arbeit werden das Vorgehen neuroradiologischer Abteilungen in Deutschland dargestellt und der Einsatz des Low-dose- Thorax-CT analysiert.

\section{Material und Methoden}

Am 08.04.2020 wurde per E-Mail-Verteiler des Berufsverbands der Deutschen Neuroradiologen (BDNR) e.V. eine Umfrage darüber gestartet, welche diagnostischen und Schutzmaßnahmen in den einzelnen Kliniken bei Patienten mit möglicher SARS-CoV-2-Infektion getroffen werden. Nach zunächst freier Mitteilung wurden die Zusender gebeten, ihr Vorgehen einer der in Tab. 2 genannten Kategorien zuzuordnen.

\section{Ergebnisse}

Bis zum 21.04.2020 haben 25 Abteilungen geantwortet; die Ergebnisse sind in Tab. 2 zusammengefasst.

Elf Abteilungen (44\%) fertigen ein Low-dose-CT des Thorax an, und zwar bei jedem Patienten $(n=3)$ oder bei Temperaturerhöhung, Risikokonstellation oder fehlender Anamnesemöglichkeit $(n=8)$. Es verlassen sich 14 Abteilungen auf die klinische Einschätzung der $\mathrm{Zu}$ weiser und legen bei COVID-19-Verdacht entsprechende Schutzkleidung an (FFP2-Maske, Schutzbrille, OP-Kittel, doppelte Schutzhandschuhe; $n=7$ ). In 2 Abteilungen wird die oben genannte Schutzkleidung nur bei positivem COVID-19-Nachweis angelegt; Mitarbeiter in 5 Abteilungen angiographieren nach Anlegen von MNS, OP-Kittel und Handschuhen.

Die Genauigkeit des Low-dose-Thorax-CT lässt sich bei den kleinen Fallzahlen RT-PCR-positiver Patienten noch nicht zuverlässig bestimmen. Erste Ergebnisse aus 5 Abteilungen sind in Tab. 3 zusammengefasst. 
Tab. 1 Vergleich der diagnostischen Genauigkeit von RT-PCR und Low-dose-Thorax-CT

\begin{tabular}{|c|c|c|c|c|c|}
\hline Studie & $\begin{array}{l}\text { RT-PCR + Low-dose-CT: } \\
n\end{array}$ & Sensitivität & Spezifität & PPV & NPV \\
\hline Ai et al. [11] & 1014, 601 RT-PCR-pos. & $97 \% ; 580 / 601$ & $\begin{array}{l}25 \% \\
105 / 413\end{array}$ & $\begin{array}{l}65 \% \\
580 / 888\end{array}$ & $\begin{array}{l}83 \% \\
105 / 126\end{array}$ \\
\hline Xie et al. [12] & 167 RT-PCR-pos. & $96 \% ; 160 / 167$ & - & - & - \\
\hline Li und Xia [13] & 53 RT-PCR-pos. & $96 \% ; 51 / 53$ & - & - & - \\
\hline Chung et al. [14] & 21 RT-PCR-pos. & $\begin{array}{l}86 \%, 18 / 21 \\
15>1 \text { Lungenlappen betroffen }\end{array}$ & - & - & - \\
\hline Bernheim et al. [15] & 121 RT-PCR-pos. & $\begin{array}{l}0-2 \text { Tage: } 44 \% ; 16 \text { von } 36 \\
\text { 3-5 Tage: } 91 \% ; 30 \text { von } 33 \\
6-12 \text { Tage: } 96 \% ; 24 \text { von } 25\end{array}$ & - & - & - \\
\hline Fang et al. [16] & 51 RT-PCR-pos. & $\begin{array}{l}98 \%, 50 / 51 \\
36 \text { typische CT-Manifestationen, } 14 \text { aty- } \\
\text { pische CT-Manifestationen } \\
\text { RT-PCR pos.: } \\
\text { Tag 0: } 36 / 51 \\
\text { Tag } 0-1: 48 / 51 \\
\text { Tag } 0-2: 50 / 51 \\
\text { Tag } 0-7: 51 / 51\end{array}$ & - & - & - \\
\hline $\begin{array}{l}\text { Wen et al. zitiert in } \\
\text { [5] }\end{array}$ & 103, 88 RT-PCR-pos. & $\begin{array}{l}93 \%, 82 / 88 \\
\text { RT-PCR pos.: } \\
\text { Tag 0-3: } 37(42 \%) \\
\text { Tag 4-5: } 66(77 \%) \\
\text { Tag 6-7: } 80(91 \%) \\
>\text { Tag 7: } 88(100 \%)\end{array}$ & $\begin{array}{l}53 \% \\
100 \% \text { per } \\
\text { definitio- } \\
\text { nem }\end{array}$ & $\begin{array}{l}92 \% \\
100 \% \text { per } \\
\text { definitio- } \\
\text { nem }\end{array}$ & $\begin{array}{l}42 \% \\
23 \%\end{array}$ \\
\hline Ling et al. [17] & 295 RT-PCR-pos. & $\begin{array}{l}0-2 \text { Tage: } 83 \% ; 246 / 295 \\
\text { 3-14 Tage: } 88 \% ; 261 / 295\end{array}$ & - & - & - \\
\hline Pan et al. [18] & 21 RT-PCR-pos. & $\begin{array}{l}0-4 \text { Tage: } 81 \% ; 17 / 21 \\
>4 \text { Tage: } 100 \% ; 21 / 21\end{array}$ & - & - & - \\
\hline Xu et al. [19] & 90 RT-PCR-pos. & $\begin{array}{l}0-2 \text { Tage: } 77 \% ; 69 / 90 \\
\text { 3-4 Tage: } 80 \% ; 72 / 90\end{array}$ & - & - & - \\
\hline Song et al. [20] & 51 RT-PCR-pos. & $\begin{array}{l}86 \% ; 44 / 51 \\
\text { Milchglastrübungen in beiden Lungen }\end{array}$ & - & - & - \\
\hline Wang et al. [21] & 90 RT-PCR-pos. & $\begin{array}{l}\text { Vor klinischen Symptomen } 40 \% ; 4 / 10 \\
\text { Tag 0-5 85\%; 60/79 } \\
\text { Tag } 7100 \% ; 1 / 1\end{array}$ & - & - & - \\
\hline Hu et al. [22] & 24 RT-PCR-pos. & Symptomfrei $71 \% ; 17 / 24$ & - & - & - \\
\hline Inui et al. [23] & 104 RT-PCR-pos. & $\begin{array}{l}61 \% ; 63 / 104 \\
\text { Symptomfrei } 54 \% ; 41 / 76 \\
\text { Symptomatisch } 79 \% ; 22 / 28\end{array}$ & - & - & - \\
\hline Chen et al. [24] & 274 RT-PCR-pos. & $97 \% ; 265 / 274$ & - & - & - \\
\hline Summe & - & $1680 / 1975=85 \%$ & - & - & - \\
\hline
\end{tabular}

$N P V$ „,negative predictive value“ (negativer prädikativer Wert), $P P V$ „positive predictive value“ (positiver prädikativer Wert), $R T-P C R$ Reverse-Transkriptase-Polymerase-Kettenreaktion

\section{Diskussion}

Wünschenswert ist, dass Patienten, bei denen eine SARSCoV-2-Infektion möglich erscheint, wie Patienten mit nachgewiesener Infektion behandelt werden [3]. Allerdings wird ein solches Vorgehen zumindest in Deutschland bei knappen Schutzmaterialien nicht flächendeckend praktiziert. Vielmehr wird versucht, eine Risikoeinschätzung vorzunehmen, die sich auf die klinische Einschätzung der Zuweiser, eine Fiebermessung und in etwa $40 \%$ der Abteilungen auf ein Low-dose-Thorax-CT stützt. Zu Low-
dose-Thorax-CT-Untersuchungen gibt es mittlerweile eine Vielzahl von Publikationen [11-24]. Problematisch ist, dass es sich fast ausschließlich um mithilfe der RT-PCR bewiesene COVID-19-Fälle handelt, sodass Aussagen zu Spezifität, positivem prädikativem Wert (,,positive predictive value“, PPV), negativem prädikativem Wert (,negative predictive value“, NPV) und Genauigkeit kaum möglich sind. Die Sensitivität des Low-dose-CT in der Frühphase der Erkrankung (0 bis 2 Tage) beträgt in retrospektiven Studien im Mittel 85\% (40-98\%; Tab. 1). Allerdings ist auch die Sensitivität der RT-PCR nicht höher; sie beträgt 
Tab. 2 Schutzmaßnahmen in deutschen neuroradiologischen Abteilungen bei der Untersuchung von Patienten mit möglicher COVID-19

\begin{tabular}{|c|c|}
\hline Multimodales CT & $\rightarrow$ Angiographie \\
\hline $\begin{array}{l}\text { MNS für den Patienten } \\
\text { Low-dose-CT-Thorax }\end{array}$ & Verdachtsfall: FFP2-Maske, Schutzbrille, OP-Kittel, doppelte Schutzhandschuhe \\
\hline $\begin{array}{l}\text { MNS für den Patienten } \\
\text { Klinische Einschätzung des Zuweisers und/oder Tem- } \\
\text { peraturerhöhung oder, wenn Temperatur- und Sympto- } \\
\text { manamnese nicht möglich: Low-dose-CT-Thorax }\end{array}$ & $\begin{array}{l}\text { Verdachtsfall: FFP2-Maske, Schutzbrille, OP-Kittel, doppelte Schutzhandschuhe } \\
\text { Lüftungsanlage wird auf Sog umgestellt }{ }^{\mathrm{a}}\end{array}$ \\
\hline $\begin{array}{l}\text { MNS für den Patienten } \\
\text { Klinische Einschätzung des Zuweisers }\end{array}$ & Verdachtsfall: FFP2-Maske, Schutzbrille, OP-Kittel, doppelte Schutzhandschuhe \\
\hline $\begin{array}{l}\text { MNS für den Patienten } \\
\text { Klinische Einschätzung des Zuweisers }\end{array}$ & $\begin{array}{l}\text { Verdachtsfall: MNS, OP-Kittel, Handschuhe } \\
\text { Anästhesist: FFP2-Maske während In- und Extubation }\end{array}$ \\
\hline MNS für den Patienten & $\begin{array}{l}\text { COVID-19-Nachweis: FFP2-Maske, Schutzbrille, OP-Kittel, doppelte Schutzhand- } \\
\text { schuhe }\end{array}$ \\
\hline
\end{tabular}

COVID-19 „coronavirus disease 2019“, FFP „,filtering face piece“, MNS Mund-Nasen-Schutz

${ }^{a}$ nicht in allen Abteilungen möglich

Tab. 3 Diagnostische Genauigkeit der Low-dose-Thorax-Computertomographie bei Patienten mit COVID-19-Verdacht

\begin{tabular}{|c|c|c|c|c|c|c|c|c|}
\hline \multirow{2}{*}{$\begin{array}{l}\text { Patienten, } \\
\text { gesamt }\end{array}$} & \multirow{2}{*}{$\begin{array}{l}\text { COVID- } \\
\text { 19-Ver- } \\
\text { dacht }\end{array}$} & \multirow{2}{*}{$\begin{array}{l}\text { Mechanische } \\
\text { Thrombek- } \\
\text { tomie }\end{array}$} & \multicolumn{2}{|l|}{ RT-PCR } & \multicolumn{4}{|c|}{ Computertomographiebefund } \\
\hline & & & Gesamt & $\begin{array}{l}\text { Positiver Be- } \\
\text { fund }\end{array}$ & Richtig-positiv & Falsch-positiv & Richtig-negativ & Falsch-negativ \\
\hline 19 & 7 & 5 & 19 & 2 & 2 & 5 & 12 & 0 \\
\hline 16 & 3 & 3 & 16 & 0 & 0 & 1 & 15 & 0 \\
\hline 3 & 0 & 3 & 3 & 0 & 0 & 0 & 3 & 0 \\
\hline 3 & 1 & 3 & 3 & 0 & 0 & 1 & 2 & 0 \\
\hline 12 & 3 & 1 & 12 & 3 & 3 & 3 & 6 & 0 \\
\hline \multicolumn{9}{|l|}{ Summe } \\
\hline 53 & 14 & 15 & 53 & 5 & 5 & 10 & 38 & 0 \\
\hline
\end{tabular}

COVID-19 „coronavirus disease 2019“, RT-PCR Reverse-Transkriptase-Polymerase-Kettenreaktion

gerade in den initialen 2 Tagen nur $60-70 \%[10,16]$ : Einige RT-PCR werden erst im Verlauf positiv, und nur wenige Patienten werden mehrfach getestet [16]. Betrachtet man symptomfreie Patienten weist die Hälfte COVID-19typische Veränderungen auf; etwa $30 \%$ der Thorax-CT-Befunde sind unauffällig [22, 23]. Ein Großteil der Patienten mit akutem Schlaganfall wird klinisch symptomfrei sein, sodass diese niedrigere Sensitivität (50-70\%) realistischer erscheint. Sich allein auf das Low-dose-CT zu verlassen, ohne zumindest eine zusätzliche Temperaturmessung vorzunehmen, ist daher problematisch.

Die Unterscheidung gegenüber anderen atypischen Pneumonien soll bei 60-83\% der Patienten gelingen [25]. Das frühzeitige Erkennen einer SARS-CoV-2-Infektion ist auch für den weiteren klinischen Verlauf von Bedeutung: Früher diagnostizierte Patienten profitieren vom besseren klinischen Verlauf [26]. Thrombektomiepatienten sind in der Mehrzahl der Fälle ältere Patienten mit Grunderkrankungen. Ältere Patienten mit COVID-19 sind schwerer erkrankt und werden 3-mal häufiger auf einer Intensivstation behandelt [2]. Sie erleiden häufiger ,,akute zerebrovaskuläre Erkrankungen (5,7 vs. 0,8\%) “ und Bewusstseinsstörungen (14,8 vs. $2,4 \%)$, sodass gerade bei akuten Erkrankungen wie einem Schlaganfall an die Möglichkeit einer SARSCoV-2-Infektion zu denken ist [27]. Für diese Patienten spielt schließlich die durch das Low-dose-CT erhöhte Strahlenexposition eine untergeordnete Rolle.

\section{Fazit für die Praxis}

- Das unterschiedliche Vorgehen neuroradiologischer Abteilungen in Deutschland spiegelt die Unsicherheit im Umgang mit Schlaganfallpatienten und möglicher ,,coronavirus disease 2019“ (COVID-19) wider.

- Ein Low-dose-CT des Thorax kann im Rahmen der multimodalen CT-Diagnostik einfach akquiriert und zur Risikoeinschätzung herangezogen werden.

- Die Limitationen hinsichtlich der Sensitivität v.a. bei symptomfreien Patienten und der Tatsache, dass ein negativer Thorax-CT-Befund eine Infektion nicht vollständig ausschließen kann, sollten beachtet werden.

Danksagung Die Autoren bedanken sich für die Rückmeldungen zum Vorgehen bei Joachim Berkefeld, Frankfurt; Ansgar Berlis, Augsburg; Tobias Boeckh-Behrens, München; Georg Bohner, Berlin; Wolfgang Bunk, Ludwigsburg; Ulrich Grzyska, Hamburg; Jörg Hattingen, Han- 
nover; Titus Hoffmann, Leipzig; Olav Jansen, Kiel; Wibke Kurre, Passau; Heiner Lanfermann, Hannover; Christian Mathys, Oldenburg; Markus Möhlenbruch, Heidelberg; Wolfgang Reith, Homburg; Stefan Rohde, Dortmund; Peter Schramm, Lübeck; László Solymosi, Bonn; Joachim Spreer, Köln; Stephanie Tritt, Wiesbaden; Bernd Turowski, Düsseldorf, Oliver Wittkugel, Buchholz

Interessenkonflikt H. Urbach, H. Janssen, J. Linn, T. Hoffmann, S. Tritt, W. Weber und M. Wiesmann geben an, dass kein Interessenkonflikt besteht.

\section{Literatur}

1. Khosravani H, Rajendram P, Notario L, Chapman MG, Menon BK. Protected code stroke hyperacute stroke management during the coronavirus disease 2019 (COVID-19) pandemic. Stroke. 2020. https://doi.org/10.1161/STROKEAHA.120.029838.

2. Li B, Yang J, Zhao F, Zhi L, Wang X, Liu L, Bi Z, Zhao Y. Prevalence and impact of cardiovascular metabolic diseases on COVID19 in China. Clin Res Cardiol. 2020;109:531-8. https://doi.org/10. 1007/s00392-020-01626-9.

3. Fraser JF, Arthur AS, Chen M, Levitt M, Mocco J, Albuquerque FC, Ansari SA, Dabus G, Jayaraman MV, Mack WJ, Milburn J, Mokin M, Narayanan S, Puri AS, Siddiqui AH, Tsai JP, Klucznik RP. Society of NeuroInterventional Surgery recommendations for the care of emergent neurointerventional patients in the setting of COVID19. J Neurointerv Surg. 2020. https://doi.org/10.1136/neurintsurg2020-016098.

4. Raptis CA, Hammer MM, Short RG, Shah A, Bhalla S, Bierhals AJ, Filev PD, Hope MD, Jeudy J, Kligerman SJ, Henry TS. Chest $\mathrm{CT}$ and Coronavirus disease (COVID-19): a critical review of the literature to date. AJR Am J Roentgenol. 2020; https://doi.org/10. 2214/AJR.20.23202

5. Simpson S, Kay FU, Abbara S, Bhalla S, Chung JH, Henry TS, Kanne JP, Kligerman Sm Ko JP, Litt H. Radiological Society of North America Expert consensus statement on reporting chest CT findings related to COVID-19. Endorsed by the Society of Thoracic Radiology, the American College of Radiology, and RSNA. 2020. https://doi.org/10.1097/RTI.0000000000000524.

6. American College of Radiology. ACR recommendations for the use of chest radiography and Computed Tomography (CT) for suspected COVID-19 infection. 2020. https://www.acr.org/Advocacyand-Economics/ACR-Position- Statements/Recommendationsfor-Chest-Radiography-and-CT-for-Suspected-COVID19-Infection. Zugegriffen: 1. Apr. 2020.

7. Society of Thoracic Radiology/American Society of Emergency Radiology COVID-19 Position Statement. 2020. https://www.acr. org/Advocacy-and-Economics/ACR-Position-Statements/Recom mendations-for-Chest-Radiography-and-CT-for-Suspected-COVID19-Infection. Zugegriffen: 22. März 2020.

8. Jin YH, Cai L, Cheng ZS, Cheng H, Deng T, Fan YP, Fang C, Huang D, Huang LQ, Huang Q, Han Y, Hu B, Hu F, Li BH, Li YR, Liang K, Lin LK, Luo LS, Ma J, Ma LL, Peng ZY, Pan YB, Pan ZY, Ren XQ, Sun HM, Wang Y, Wang YY, Weng H, Wei CJ, Wu DF, Xia J, Xiong Y, Xu HB, Yao XM, Yuan YF, Ye TS, Zhang XC, Zhang YW, Zhang YG, Zhang HM, Zhao Y, Zhao MJ, Zi H, Zeng XT, Wang YY, Wang XH; , for the Zhongnan Hospital of Wuhan University Novel Coronavirus Management and Research Team, Evidence-Based Medicine Chapter of China International Exchange and Promotive Association for Medical and Health Care (CPAM). A rapid advice guide-line for the diagnosis and treatment of 2019 novel coronavirus (2019-nCoV) infected pneumonia (standard version). Mil Med Res. 2020;7:4.

9. Rubin GD, Ryerson CJ, Haramati LB, Sverzellati N, Kanne JP, Raoof S, Schluger NW, Volpi A, Yim JJ, Martin IBK, Anderson DJ,
Kong C, Altes T, Bush A, Desai SR, Goldin J, Goo JM, Humbert M, Inoue Y, Kauczor HU, Luo F, Mazzone PJ, Prokop M, RemyJardin M, Richeldi L, Schaefer-Prokop CM, Tomiyama N, Wells $\mathrm{AU}$, Leung AN. The role of chest imaging in patient management during the COVID-19 pandemic: a multinational consensus statement from the Fleischner Society. Radiology. 2020. https://doi.org/ 10.1148/radiol.2020201365.

10. Antoch G, Urbach H, Mentzel HJ, Reimer P, Weber W, Wujciak D. SARS-CoV-2/COVID-19: Empfehlungen für die Radiologische Versorgung - Eine Stellungnahme der Deutschen Röntgengesellschaft (DRG), der Deutschen Gesellschaft für Neuroradiologie (DGNR), der Gesellschaft für Pädiatrische Radiologie (GPR), der Deutschen Gesellschaft für Interventionelle Radiologie (DeGIR), des Berufsverbands der Neuroradiologen (BDNR) und des Berufsverbands der Radiologen (BDR). Rofo. 2020;192:418-21. https:// doi.org/10.1055/a-1149-3625.

11. Ai T, Yang Z, Hou H, Zhan C, Chen C, Lv W, Tao Q, Sun Z, Xia L. Correlation of chest CT and RT-PCR testing in coronavirus disease 2019 (COVID-19) in China: a report of 1014 cases. Radiology. 2020. https://doi.org/10.1148/radiol.2020200642.

12. Xie X, Zhong Z, Zhao W, Zheng C, Wang F, Liu J. Chest CT for typical 2019-nCoV pneumonia: relationship to negative RT-PCR testing. Radiology. 2020. https://doi.org/10.1148/radiol.2020200343.

13. Li Y, Xia L. Coronavirus disease 2019 (COVID-19): role of chest CT in diagnosis and management. AJR Am J Roentgenol. 2020; https://doi.org/10.2214/AJR.20.22954.

14. Chung M, Bernheim A, Mei X, Zhang N, Huang M, Zeng X, Cui J, Xu W, Yang Y, Fayad ZA, Jacobi A, Li K, Li S, Shan H. CT imaging features of 2019 novel coronavirus (2019-nCoV). Radiology. 2020;295:202-7. https://doi.org/10.1148/radiol.2020200230.

15. Bernheim A, Mei X, Huang M, Yang Y, Fayad ZA, Zhang N, Diao K, Lin B, Zhu X, Li K, Li S, Shan H, Jacobi A, Chung M. Chest CT findings in coronavirus disease-19 (COVID-19): relationship to duration of infection. Radiology. 2020. https://doi.org/10.1148/radiol. 2020200463.

16. Fang Y, Zhang H, Xie J, Lin M, Ying L, Pang P, Ji W. Sensitivity of chest CT for COVID-19: comparison to RT-PCR. Radiology. 2020. https://doi.org/10.1148/radiol.2020200432.

17. Ling Z, Xu X, Gan Q, Zhang L, Luo L, Tang X, Liu J. Asymptomatic SARS-CoV-2 infected patients with persistent negative CT findings. Eur J Radiol. 2020;126:108956. https://doi.org/10.1016/j. ejrad.2020.108956.

18. Pan F, Ye T, Sun P, Gui S, Liang B, Li L, Zheng D, Wang J, Hesketh RL, Yang L, Zheng C. Time course of lung changes on chest CT during recovery from 2019 novel coronavirus (COVID19) pneumonia. Radiology. 2020. https://doi.org/10.1148/radiol. 2020200370.

19. Xu X, Yu C, Qu J, Zhang L, Jiang S, Huang D, Chen B, Zhang Z, Guan W, Ling Z, Jiang R, Hu T, Ding Y, Lin L, Gan Q, Luo L, Tang X, Liu J. Imaging and clinical features of patients with 2019 novel coronavirus SARS-CoV-2. Eur J Nucl Med Mol Imaging. 2020;47:1275-80. https://doi.org/10.1007/s00259-020-047359.

20. Song F, Shi N, Shan F, Zhang Z, Shen J, Lu H, Ling Y, Jiang Y, Shi Y. Emerging 2019 novel coronavirus (2019-nCoV) pneumonia. Radiology. 2020;295:210-7. https://doi.org/10.1148/radiol. 2020200274.

21. Wang Y, Dong C, Hu Y, Li C, Ren Q, Zhang X, Shi H, Zhou M. Temporal changes of CT findings in 90 patients with COVID-19 pneumonia: a longitudinal study. Radiology. 2020. https://doi.org/ 10.1148/radiol.2020200843.

22. Hu Z, Song C, Xu C, Jin G, Chen Y, Xu X, Ma H, Chen W, Lin Y, Zheng Y, Wang J, Hu Z, Yi Y, Shen H. Clinical characteristics of 24 asymptomatic infections with COVID-19 screened among close contacts in Nanjing, China. Sci China Life Sci. 2020;63:706-11. https://doi.org/10.1007/s11427-020-1661-4. 
23. Inui S, Fujikawa A, Jitsu M, Kunishima N, Watanabe S, Suzuki Y, Umeda S, Uwabe Y. Chest CT findings in cases from the cruise ship "Diamond Princess" with Coronavirus disease 2019 (COVID19). Radiol Cardiothorac Imaging. 2020. https://doi.org/10.1148/ ryct.2020200110.

24. Chen T, Wu D, Chen H, Yan W, Yang D, Chen G, Ma K, Xu D, Yu H, Wang H, Wang T, Guo W, Chen J, Ding C, Zhang X, Huang J, Han M, Li S, Luo X, Zhao J, Ning Q. Clinical characteristics of 113 deceased patients with coronavirus disease 2019: retrospective study. BMJ. 2020;368:m1091. Erratum in: BMJ. 2020;368:m1295. https://doi.org/10.1136/bmj.m1091.

25. Bai HX, Hsieh B, Xiong Z, Halsey K, Choi JW, Tran TML, Pan I, Shi LB, Wang DC, Mei J, Jiang XL, Zeng QH, Egglin TK, Hu PF, Agarwal S, Xie F, Li S, Healey T, Atalay MK, Liao WH. Performance of radiologists in differentiating COVID-19 from viral pneu- monia on chest CT. Radiology. 2020. https://doi.org/10.1148/radiol. 2020200823.

26. Huang G, Gong T, Wang G, Wang J, Guo X, Cai E, Li S, Li X, Yu Y, Lin L. Timely diagnosis and treatment shortens the time to resolution of coronavirus disease (COVID-19) pneumonia and lowers the highest and last CT scores from sequential chest CT. AJR Am J Roentgenol. 2020. https://doi.org/10.2214/AJR.20.23078.

27. Mao L, Jin H, Wang M, Hu Y, Chen S, He Q, Chang J, Hong C, Zhou Y, Wang D, Miao X, Li Y, Hu B. Neurologic manifestations of hospitalized patients with coronavirus disease 2019 in Wuhan, China. JAMA Neurol. 2020. https://doi.org/10.1001/jamaneurol.2020. 1127. 